\title{
Prevalence and Characteristics of hrHPV Infection among 414,540 Women: A Multicenter Study in Central and Eastern China
}

\author{
Yi Wang ${ }^{1^{*}}$, Yifan Meng 2,11*, Wending Li $1^{*}$, Xiaofei Zhang ${ }^{3}$, Zaixing Deng ${ }^{4}$, Mengjun Hu ${ }^{5}$, Pingrong Shen 6 , \\ Shengfeng $\mathrm{Xu}^{7}$, Chenglin $\mathrm{Fu}^{8}$, Wen Jiang ${ }^{9}$, Bing Wu ${ }^{10}$, Kezhen $\mathrm{Li}^{2,11}$, Gang Chen 2,11, Juncheng Wei 2,11, \\ Ling $\mathrm{Xi}^{2,11}$, Junbo $\mathrm{Hu}^{11}$, Xing Xie ${ }^{3}$, Ding Ma ${ }^{2,11}$, Xiaodong Cheng ${ }^{3 凶}$, Peng Wu ${ }^{2,11 \bowtie}$ \\ 1. School of Public Health, Tongji Medical College, Huazhong University of Science and Technology, Wuhan, Hubei, China \\ 2. Department of Gynecologic Oncology, Tongii Hospital, Tongii Medical College, Huazhong University of Science and Technology, Wuhan, Hubei, China \\ 3. Department of Gynecologic Oncology, Women's Hospital, School of Medicine, Zhejiang University, Hangzhou, Zhejiang, China \\ 4. Department of Obstetrics and Gynecology, Huzhou Maternity and Child Care Center, Huzhou, Zhejiang, China \\ 5. Department of Obstetrics and Gynecology, Zhuji People's Hospital, Shaoxing, Zhejiang, China \\ 6. Department of Obstetrics and Gynecology, Ninghai Maternity and Child Care Center, Ningbo, Zhejiang, China \\ 7. Department of Obstetrics and Gynecology, Jiaxing Maternity and Child Care Center, Jiaxing, Zhejiang, China \\ 8. Department of Obstetrics and Gynecology, Taizhou First People's Hospital, Taizhou, Zhejiang, China \\ 9. Department of Obstetrics and Gynecology, Zhoushan Maternity and Child Care Center, Zhoushan, Zhejiang, China \\ 10. Department of Obstetrics and Gynecology, Zhuji Maternity and Child Care Center, Shaoxing, Zhejiang, China \\ 11. The Key Laboratory of Cancer Invasion and Metastasis of the Ministry of Education of China, Tongji Hospital, Tongji Medical College, Huazhong \\ University of Science and Technology, Wuhan, Hubei, China \\ ${ }^{*}$ Co-first authors
}

$\square$ Corresponding authors: Peng Wu, Address: Department of Gynecologic Oncology, Tongii Hospital, Tongii Medical College, Huazhong University of Science and Technology, Wuhan, Hubei, China. The Key Laboratory of Cancer Invasion and Metastasis of the Ministry of Education of China, Tongji Hospital, Tongji Medical College, Huazhong University of Science and Technology, Wuhan, Hubei, China. Phone: +86-13995573729. Fax: 027-83662681. Email: pengwu8626@126.com or Xiaodong Cheng, Address: Department of Gynecologic Oncology, Women's Hospital, School of Medicine, Zhejiang University, Hangzhou, Zhejiang, China. Phone: +86-13958039839. Email: chengxd@zju.edu.cn.

(c) Ivyspring International Publisher. This is an open access article distributed under the terms of the Creative Commons Attribution (CC BY-NC) license (https://creativecommons.org/licenses/by-nc/4.0/). See http://ivyspring.com/terms for full terms and conditions.

Received: 2018.09.24; Accepted: 2019.02.27; Published: 2019.04.21

\begin{abstract}
Objectives: Understanding the prevalence and characteristics of high-risk human papillomavirus (hrHPV) with the large-scale multicenter data based on a US FDA-approved testing method is important to guide ongoing vaccination programs in China.

Methods: We conducted a retrospective observational study based on data from 11 large hospitals in central and eastern China. From October 1st, 2012 to December 31st, 2016, a total of 480,034 cervical specimens were collected, and 414,540 eligible participants (14-80 years, mean age 39.9 years) were included and tested using Cervista High-Risk HPV Assay (Hologic Inc., Bedford, Mass, USA).

Results: The overall hrHPV prevalence in this study was $17.8 \%(73,713 / 414,540)$, with Wuhan slightly higher than Zhejiang ( $18.6 \%$ vs. $17.6 \%, \mathrm{P}<0.001)$. The prevalence showed a declining trend from 2012 to 2016. The most common hrHPV group was A9 (61.7\%), followed by A5/A6 (29.4\%) and A7 (25.6\%). A U-shaped curve was observed for age-specific hrHPV prevalence: $\leq 19$ years and $\geq 50$ years were higher than other age groups.

Conclusion: In pre-vaccination period, A9 was the most dominant hrHPV group, and infections were most likely to occur at younger and older ages. The prevalence of hrHPV varied by cities and age groups, suggesting vaccination programs should be propagated in a population-specific approach.
\end{abstract}

Key words: Cervista, China, distribution, high-risk human papillomavirus, multicenter, prevalence 


\section{Introduction}

As one of the most serious health concerns to women all around the world, cervical cancer ranks second in all diagnosed cancers in women from developing countries.[1] Almost all cases of cervical cancers are caused by persistent infection with high-risk human papillomavirus (hrHPV), including HPV types 16, 18, 31, 33, 35, 39, 45, 51, 52, 56, 58, 59, 66, and 68.[2] Early hrHPV screening of the general population is essential for early detection and intervention of CIN and cervical cancers. According to a meta-analysis that studied 1 million women with negative cytology results, the estimated global HPV prevalence was $11.7 \%$.[3] Even though several studies have assessed the prevalence of HPV strains in mainland China,[4-10] most of them focused on HPV genotyping test approved by the National Medical Products Administration (NMPA). So far, NMPA has approved more than 100 HPV DNA test kits [11] with only five high-risk (HR) HPV DNA test kits approved by U.S. Food and Drug Administration (USFDA) since 2003. To unveil HPV prevalence patterns in central and eastern China, we recorded 480,034 tests using Cervista High-Risk HPV Assay (Hologic Inc., Bedford, Mass, USA) in eight large cities in China. Cervista HPV HR is widely used in primary cervical screening in China. It can detect the 14 types of HPV grouped according to phylogenetic relatedness, i.e., viral types with similar DNA sequences. These probe sets are A5/ A6 (HPV types 51, 56, and 66), A7 (HPV types $18,39,45,59$, and 68 ), and A9 (HPV types 16, 31, $33,35,52$, and 58 ).

\section{Materials and Methods}

\section{Study population}

This study was based on HPV test results obtained from 11 large hospitals located in 2 districts in central and eastern China. Three hospitals are located in the Wuhan district and eight in the Zhejiang district. (See Table S1 for more details) From October 1st, 2012 to December 31st, 2016, a total of 480,034 cervical specimens were collected at hospital-affiliated gynecological outpatient clinics and test centers. The selection criteria were as follows: (1) had a history of sexual activity; (2) were not pregnant or using vaginal medications at the time of enrolment; (3) had no history of total hysterectomy or cervical resection; (4) provided written consent to undergo HPV testing. Details on the selection process have been described by using flow chart (Figure 1). Information pertaining to HPV DNA tests was obtained from medical archives. This study was approved by the Ethical Committee of Tongji Hospital (TJ-IRB20171001), Tongji Medical College, Huazhong
University of Science and Technology, Wuhan, China. The investigation procedure was carried out in accordance with the approved guidelines and the principles expressed in the Declaration of Helsinki.

\section{Cervical specimen collection}

Cervicovaginal swabs were collected from all participants by a gynecologist according to the standard operation procedure for sampling at the recruitment sites. Cervical specimens were collected in PreservCyt ${ }^{\circledR}$ Solution, the ThinPrep ${ }^{\circledR}$ Pap Test preservation system, using a broom-type device (e.g., Rovers Cervex ${ }^{\circledR}$ Brush, Wallach Papette $\left.{ }^{\circledR}\right)$, or Endocervical Brush/Spatula. The cervical specimens were stored at room temperature $\left(20-30^{\circ} \mathrm{C}\right)$ in PreservCyt ${ }^{\circledR}$ Solution prior to testing. All samples were shipped to the lab for HPV testing within 24 hours.

\section{HPV groups}

The Cervista HPV HR test used the Invader ${ }^{\circledR}$ chemistry, a signal amplification method for detection of specific nucleic acid sequences.[12] Based on the correlation among 14 types of hrHPV DNA gene sequences, the $14 \mathrm{HPV}$ DNA types were tested in groups with three different oligonucleotide mixtures, A5/A6, A7, and A9. An A5/A6 oligonucleotide probe mixture (Oligo Mixes) was used for detecting HPV types 51,56, and 66 (the A5/ A6 group of HPV), an A7 oligonucleotide probe mixture was used for HPV types $18,39,45,59$, and 68 (the A7 group of HPV), and an A9 oligonucleotide mixture was used for HPV types of $16,31,33,35,52$, and 58 (the A9 group of HPV).

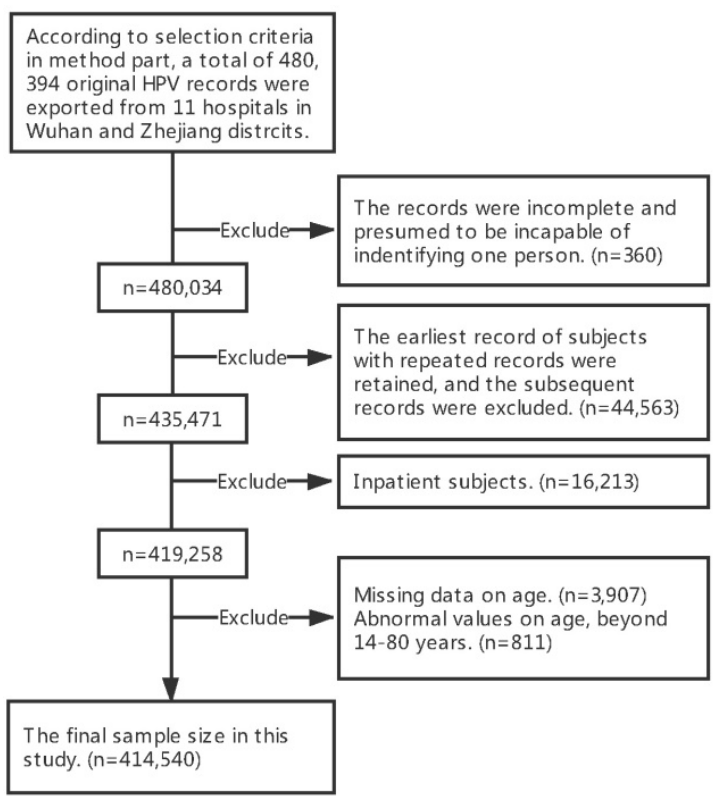

Figure 1. Subjects selection flow chart. 


\section{Data analysis}

Numerical data were summarized as means \pm standard deviation (SD) and categorical variables were presented as percentage (\%). The overall and age-specific hrHPV prevalence were calculated by dividing positive cases by total or age-specific subjects. The hrHPV group-specific and single/multiple-group prevalence were calculated as the percentages of corresponding positive cases within total positive cases. Two-sided $95 \%$ confidence interval $(95 \% \mathrm{CI})$ was estimated for each calculation. We further stratified the data by age ( $\leq 19$ years, 20-29 years, 30-39 years, 40-49 years, and $\geq 50$ years), specific Cervista groups and regions, and tested statistical significance using Chi-square tests. Raw data were entered into an Excel spread sheet and analyzed using SPSS 22.0 statistical software. $P<0.05$ (2-tailed) was considered statistically significant.

\section{Results}

This study included 480,034 hospital visits with a mean age of $39.9 \pm 10.4$ years (Zhejiang district, $40.2 \pm$ 10.4 years; Wuhan district, $38.1 \pm 10.1$ years). As displayed in Table 1, the overall hrHPV prevalence in this study was $17.8 \%(73,713 / 414,540)$, with the Wuhan district presenting a slightly higher rate than the Zhejiang district $(18.6 \%$ vs. $17.6 \% ; P<0.001)$. However, great variability in hrHPV prevalence rate was observed among eight cities (14.0\%-20.6\%). hrHPV single-group infection accounted for $83.6 \%$ positive tests, and multiple-group accounted for $16.4 \%$. The prevalence of single-group within hrHPV positive tests were higher than that of multiple-group in all cities. We also presented the prevalence of hrHPV by year in Figure 2. hrHPV prevalence seemed to have a declining trend from 2012 to 2016. ( $P$ for trend $<0.001$ )

Table 2 presents the distribution of hrHPV group infection. The percentage of the A9 group infection was higher than that of the A5/A6 group and the A7 group $(61.7 \%$ of positive cases and $11.1 \%$ of all subjects). Single-group infection (83.6\% of positive cases and $15.1 \%$ of all subjects) more common than multiple-group infection. The most prevalent hrHPV group, in either single or multiple-group infection, was the A9 group. Of single-group infection, $60.8 \%$ and $20.8 \%$ were from the A9 and A5/A6 groups respectively. Moreover, of multiple-group infection, $66.3 \%$ and $73.3 \%$ were from the A9 and A5/ A6 groups respectively (Table S2). The proportion of the A5/A6 group, A9 group and multiple-group infection in hrHPV positive cases in the Wuhan district were higher than that in the Zhejiang district $(31.2 \%$ vs. $28.9 \%, 64.3 \%$ vs. $60.9 \%, 19.8 \%$ vs. $\left.15.3 \%, P_{s}<0.001\right)$.
Table 3 and Figure 3A show the distribution of hrHPV infection subdivided into five age groups. In the study population, the age-specific prevalence appeared to be U-shaped with $\leq 19$ years $(28.2 \%, 95 \%$ CI: $25.6-30.7)$ and $\geq 50$ years (21.4\%, 95\% CI: $21.1-21.7)$ groups exhibiting higher prevalence than any other age group (all $P_{\mathrm{s}}<0.001$, Table S3). Among hrHPV positive cases, A5/A6, A7, and A9 groups accounted for $29.4 \%, 25.6 \%$ and $61.7 \%$ respectively. A9 was the most common HPV group in each age group of women. The proportion of single-group infection in hrHPV positive was highest at $30-49$ years $(85.65 \%)$, while multiple-group was highest in the $\leq 19$ and $\geq 50$ years groups $(25.2 \%$ and $21.1 \%$, respectively). Age-specific hrHPV prevalence varied by regions. Women from Zhejiang district were more likely to contract hrHPV at a younger age ( $<30$ years), while after 30 years of age, hrHPV infection was more common among women from the Wuhan district (Figure 3B). Additionally, the multiple-group infection proportion was higher in the Wuhan district than in the Zhejiang district except $\leq 19$ years group (Figure S1).

\section{Discussion}

This study depicted the prevalence of hrHPV in Central and Eastern China from 8 municipalities. These regions do not share geographical boarders, thus representing totally different regions in this large country. Among all 414,540 tests, 17.8\% (73,713/ $414,540)$ presented hrHPV positive, while reported hrHPV prevalence among Chinese populations varied from $16.51 \%-30.90 \% .[4,6,7,13,14]$ It is worth noting that there are 6182 tests from women aged $<21$ years and 99,390 tests from women aged $<30$ years in the current study. In most clinical guidelines, HPV DNA testing was recommended to include co-testing with cervical cytology in screening only for women $\geq 30$ years, while women aged $<21$ years are not advised to undergo cervical cancer screening.[15, 16] Therefore, most of these 99,390 tests are unruly and reflect the non-standardized cervical screening protocol in mainland China. It is necessary to regulate the specific scope of application and standard processes of HPV screening. Nevertheless, these data is valuable for tracking HPV epidemics, and its infection and immune mechanisms in teenagers. Furthermore, the lack of nationwide cervical cancer screening in China makes prophylactic vaccinations particularly important. The evidence of HPV prevalence in teenagers is essential and timely in light of the forthcoming implementation of national vaccination programs, especially for determining the optimal age for HPV vaccination in China. 
Table 1. Prevalence of hrHPV infections in 8 cities of central and eastern China: 2012-2016

\begin{tabular}{|c|c|c|c|c|c|}
\hline Region & Total people & Positive records & Prevalence $(\%)(95 \% \mathrm{CI})$ & Single-group infection $(\%)^{a}$ & Multiple-group infection (\%)a \\
\hline \multicolumn{6}{|c|}{ Wuhan district ${ }^{b}$} \\
\hline Wuhan & 76,513 & 14,268 & $18.6(18.4,18.9)$ & $80.2(79.5,80.8)$ & $19.8(19.2,20.5)$ \\
\hline \multicolumn{6}{|c|}{ Zhejiang districtc } \\
\hline Hangzhou & 76,293 & 13,908 & $18.2(18.0,18.5)$ & $87.3(86.8,87.9)$ & $12.7(12.1,13.2)$ \\
\hline Huzhou & 47,329 & 6,622 & $14.0(13.7,14.3)$ & $86.7(85.9,87.5)$ & $13.3(12.5,14.1)$ \\
\hline Jiaxing & 69,265 & 11,535 & $16.7(16.4,16.9)$ & NA & NA \\
\hline Ninghai & 36,269 & 6,117 & $16.9(16.5,17.3)$ & $85.0(84.1,85.9)$ & $15.0(14.1,15.9)$ \\
\hline Taizhou & 31,855 & 5,470 & $17.2(16.8,17.6)$ & $86.9(86.0,87.8)$ & $13.1(12.2,14.0)$ \\
\hline Zhoushan & 19,094 & 3,925 & $20.6(20.0,21.1)$ & $83.7(82.6,84.9)$ & $16.3(15.1,17.4)$ \\
\hline Zhuji & 57,922 & 11,868 & $20.1(20.2,20.8)$ & $79.5(78.8,80.2)$ & $20.5(19.8,21.2)$ \\
\hline Subtotal & 338,027 & 59,445 & $17.6(17.5,17.7)$ & $84.7(84.3,85.0)$ & $15.3(15.0,15.7)$ \\
\hline Total & 414,540 & 73,713 & $17.8(17.7,17.9)$ & $83.6(83.3,83.9)$ & $16.4(16.1,16.7)$ \\
\hline
\end{tabular}

Abbreviations: NA, not available. a Single-group and multiple-group infection $(\%)=$ corresponding number of cases / hrHPV positive people * 100 ; ${ }^{~}$ Three hospitals from the

Wuhan district; ${ }^{c}$ Eight hospitals from the Zhejiang district. Chi-Square analyses of hrHPV prevalence between the two districts: $x^{2}=48.2, P<0.001$.

Table 2. Distribution of hrHPV infection groups in study population

\begin{tabular}{|c|c|c|c|c|c|}
\hline & \multicolumn{5}{|l|}{ hrHPV infection } \\
\hline & A5/A6 group & A7 group & A9 group & single-group & multiple-group \\
\hline Total cases & 18,276 & 15,931 & 38,374 & 51,999 & 10,179 \\
\hline \multicolumn{6}{|l|}{ Prevalence,$\%$} \\
\hline among positive cases & $29.4(29.0,29.8)$ & $25.6(25.3,26.0)$ & $61.7(61.3,62.1)$ & $83.6(83.3,83.9)$ & $16.4(16.1,16.7)$ \\
\hline among total women & $5.3(5.2,5.4)$ & $4.6(4.5,4.7)$ & $11.1(11.0,11.2)$ & $15.1(14.9,15.2)$ & $2.9(2.9,3.0)$ \\
\hline Wuhan district, \% (95\% CI) (among positive cases) & $31.2(30.4,31.9)$ & $25.2(24.5,26.0)$ & $64.3(63.6,65.1)$ & $80.2(79.5,80.8)$ & $19.8(19.2,20.5)$ \\
\hline Zhejiang district, \% (95\% CI) (among positive cases) & $28.9(28.5,29.3)$ & $25.7(25.3,26.1)$ & $60.9(60.5,61.4)$ & $84.7(84.3,85.0)$ & $15.3(15.0,15.7)$ \\
\hline$P$ value & $<0.001$ & 0.161 & $<0.001$ & $<0.001$ & $<0.001$ \\
\hline
\end{tabular}

a $P$ value was calculated by Chi-Square analysis between two districts. The total number of cases with A5/A6, A7 and A9 groups is not equal to the total positive records because of one person might have more than one group infected. The data of Jiaxing Maternity and Child Health Care Hospital was excluded because the group-specific information was not available.

Table 3. Distribution of hrHPV infection in study participants by age groups (except jiaxing)

\begin{tabular}{|c|c|c|c|c|c|c|}
\hline & \multicolumn{6}{|c|}{ Age group (years) } \\
\hline & $\leq 19$ & $20-29$ & $30-39$ & $40-49$ & $\geq 50$ & All \\
\hline Total cases $(\%)$ & $1,225(0.3)$ & $64,536(18.7)$ & $106,636(30.9)$ & $113,953(33.0)$ & 58,925 (17.1) & $345,275(100.0)$ \\
\hline hrHPV positive cases & 345 & 10,962 & 18,233 & 20,031 & 12,607 & 62,178 \\
\hline Prevalence, $\%(95 \% \mathrm{CI})$ & $28.2(25.6,30.7)$ & $17.0(16.7,17.3)$ & $17.1(16.9,17.3)$ & $17.6(17.4,17.8)$ & $21.4(21.1,21.7)$ & $18.0(17.9,18.1)$ \\
\hline \multicolumn{7}{|l|}{ hrHPV group, \% } \\
\hline $\mathrm{A} 5 / \mathrm{A} 6$ & $33.3(28.4,38.3)$ & $29.6(28.7,30.4)$ & $28.9(28.2,29.5)$ & $28.3(27.6,28.9)$ & $31.6(30.8,32.5)$ & $29.4(29.0,29.8)$ \\
\hline A7 & $31.3(26.4,36.2)$ & $26.2(25.4,27.1)$ & $24.7(24.1,25.3)$ & $24.9(24.3,25.5)$ & $27.4(26.6,28.2)$ & $25.6(25.3,26.0)$ \\
\hline A9 & $61.2(56.0,66.3)$ & $61.9(61.0,62.8)$ & $61.2(60.5,61.9)$ & $61.6(60.9,62.3)$ & $62.5(61.7,63.4)$ & $61.7(61.3,62.1)$ \\
\hline \multicolumn{7}{|c|}{ Multiplicity of hrHPV group infection, $\%$} \\
\hline Single-group & $74.8(70.2,79.4)$ & $82.6(81.9,83.3)$ & $85.5(85.0,86.0)$ & $85.6(85.1,86.1)$ & $78.9(78.2,79.6)$ & $83.6(83.3,83.9)$ \\
\hline Multiple-group & $25.2(20.6,29.8)$ & $17.4(16.7,18.1)$ & $14.5(14.0,15.0)$ & $14.4(13.9,14.9)$ & $21.1(20.4,21.8)$ & $16.4(16.1,16.7)$ \\
\hline
\end{tabular}

A7 (\%), A9 (\%), A5/ A6 (\%), Single-group infection (\%), Multiple-group infection (\%):calculated within hrHPV positive cases. The data of Jiaxing Maternity and Child Health Care Hospital was excluded because the group-specific information was not available.

The prevalence of hrHPV gradually decreased from 2012 to 2016. HPV DNA testing was recommended as a triage-reflex testing to further evaluate women with an atypical squamous cells of undetermined significance (ASC-US) cytology result in 2012.[17] Primary hrHPV screening alone was recommended as an alternative to current screening method in 2015.[16] Therefore, as the notable advancement in screening strategy and laboratory testing, the use of hrHPV DNA tests were expanded. Our data also suggested that the number of tested patients involved in the study has mushroomed over time. The annual visits increased tenfold from 2013 to 2016 , and the exact visit numbers were 15,443, 101,309, 152,714, and 144,171 for 2013-2016, respectively. This trend indicated that the coverage of HPV DNA tests amplified remarkably and the market share of Cervista increased over time. The observed decline could be explained by either the improvement of the quality assurance/quality control for true positive diagnosis or the mixture of primary screening samples and opportunistic samples. Furthermore, the economic development and enhanced women's health awareness might have also contributed to the increase.

A U-shaped hrHPV prevalence curve across different age groups was observed, with the prevalence peaking in the $\leq 19$ and $\geq 50$ age groups and staying relatively low through $20-49$ years, which is consistent with the trend that has been observed in 
other developing countries.[3, 6, 18] The first peak of HPV infection occurred within the younger age group just after the beginning of sexual activity. Younger women are sensitive to HPV because of the immature immune protection for HPV and frequent sexual intercourse. Zhao et al. [19] conducted a multicenter survey on age of sexual debut and sexual behavior in Chinese women and concluded a trend towards earlier sexual debut and riskier sexual behaviors. The second peak appeared in the $\geq 50$-year-old group. This trend reflected the viral persistence or reactivation of latent HPV due to higher viral loads, higher proportion of multiple-group infection and higher load of integrated HPV16 caused by physiological and immunological disorders related to hormone fluctuations during menopausal transition.[20] Since other studies have claimed that HPV incidence gradually declines with increasing age in developing countries, [3] this kind of difference may come from discrepancy in screening strategies and socioeconomic development. Nonetheless, the exact mechanisms involved in the progression of HPV prevalence require more well-designed studies for validation.

The study also compared the hrHPV prevalence in women residing in Wuhan district and Zhejiang district, which are located in central and eastern China. Although women in both districts displayed similar age-specific infection patterns, the fluctuation tendency of HPV prevalence was somewhat different across geographical regions. For the Zhejiang district, younger women aged $\leq 19$ years seemed to have the highest hrHPV prevalence $(28.2 \%)$, whereas the highest prevalence for the Wuhan district $(21.4 \%)$ appeared in older women aged $\geq 50$ years. The overall prevalence of hrHPV infection in women in the Wuhan district was slightly higher than that in the Zhejiang district (18.6\% vs. $17.6 \%)$. In addition, the proportion of A9 group and multiple-group infection in hrHPV positives of the Wuhan district were higher than that of the Zhejiang district. The regional discrepancy of hrHPV prevalence may be attributed to different economic levels, lifestyle habits, and individual awareness of HPV screening in the two districts. Since the incidence of cervical cancer in the Wuhan district $(27.1 / 100,000)$ was higher than that of Zhejiang $(11.0 / 100,000),[21]$ the distribution of cervical cancer is consistent with the present hrHPV prevalence of these two districts. Moreover, the prevalence of sexual intercourse was higher among teenagers of the Zhejiang district than that of the Wuhan district,[22] which also contributed to the higher hrHPV prevalence in the <19-year-old group of Zhejiang, though the confidence intervals were both too wide in this group.

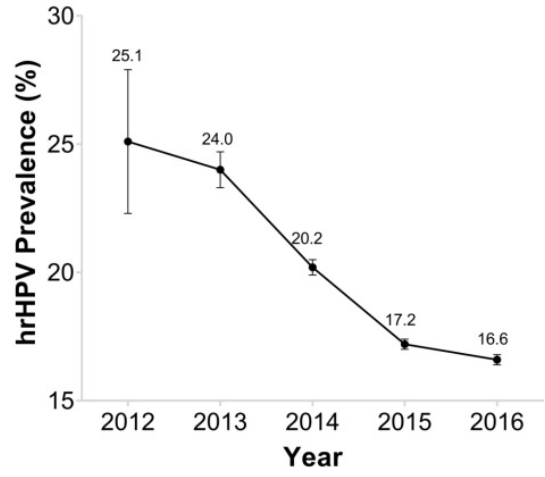

Figure 2. hrHPV prevalence by year in 8 cities of central and eastern China: 2012-2016.
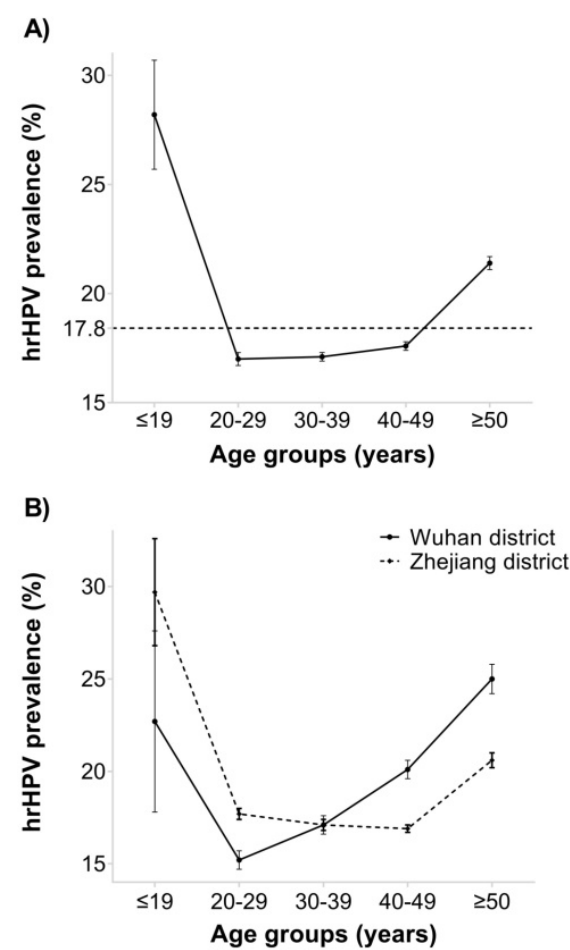

Figure 3. Prevalence of age-specific hrHPV infection and corresponding $95 \%$ confidence intervals in study population. (A) Overall age-specific hrHPV prevalence: significant difference in $\leq 19$ years and $\geq 50$ years compared with any other age group ( $P s<0.001$ ), (B) Comparison of age-specific hrHPV infection between two regions (Wuhan district vs. Zhejiang district): significant difference in age-groups of $20-39,40-49$ and $\geq 50\left(P_{s}<0.001\right)$.

In this study, the A9 HPV group, which included HPV types of $16,31,33,35,52$, and 58, accounted for $61.7 \%$ of all hrHPV positives and $11.1 \%$ of all tested people. This group was significantly greater than that of the A5/A6, A7 groups. When comparing HPV groups by age, the A9 group was the most prevalent, similar to other reports from China and other Asian regions.[4, 5, 9, 23] The A9 group had the highest proportion of women with hrHPV positive aged 40-49 years old and the lowest for $\leq 19$ year-old women. Differences among the relative hrHPV groups infection might be related to the geographical and biological interactions between various groups, and 
the host immunological characteristics.[24] The infection of A9 HPV group may show the latent persistence tendency, especially for HPV type 16, the most common HPV genotype in high grade cervical intraepithelial neoplasia (CIN) and cervical cancer. A related study also proposed that using the Cervista A9 alone as the primary screening had reasonable sensitivity and specificity for CIN 2.[25]

The advent of the prophylactic HPV vaccines is a significant public health milestone.[26] However, they were previously unavailable in mainland China until the recent licensing of the bivalent HPV vaccine which covers HPV 16 and 18, and the tetravalent HPV vaccine which covers HPV 16, 18, 6, and 11. Therefore, the current study may provide necessary information for related cost-effective analysis and regional and age-oriented vaccination programs in central and eastern China. Furthermore, the Cervista HPV DNA test was approved by the US FDA and recommended for clinical use in published guidelines, which makes our data more reliable, especially with a considerable database containing nearly half a million visit counts. $[15,16,27]$ On the other hand, a comprehensive characterization of the infection status quo will provide both the Chinese government and other stakeholders with the information needed to tailor screening strategies and interventions. However, this analysis of HPV infection has also encountered several potential limitations. First, due to lack of organized cervical cancer screening program in mainland China, some samples recruited in the study came from women with opportunistic cervical lesions. Secondly, the exact prevalence may be biased because of the absence of supporting cytological data and sampling of tissue specimens. Nevertheless, large amounts of data were gathered to overcome these shortcomings. Thirdly, Cervista assay cannot distinguish the exact genotypes, and failed to show the estimated genotype-specific infections. Further analysis with cytological data and tissue specimens is planned.

To the best of our knowledge, this is the largest routine clinical practice reports on HPV prevalence based on a US FDA-approved test method. These findings may help to estimate the burden of hrHPV infection overall and in different age groups, provide necessary information for cost-effective analysis and help in assessing the efficacy of vaccination programs in the future.

\section{Abbreviations}

CI: confidence interval; hrHPV: high-risk human papillomavirus; OR: odds ratio; SD: standard deviation.

\section{Supplementary Material}

Supplementary figures and tables.

http://www.jcancer.org/v10p1902s1.pdf

\section{Acknowledgements}

We appreciate Cordelle Lazare (Huazhong University of Science and Technology), and Rajluxmee Beejadhursing (Huazhong University of Science and Technology) for their contributions in manuscript editing.

\section{Funding}

This work was supported by Natural Science Foundation of China (81630060, 81772775), National Basic Research Program (973 Program) from Ministry of Science and Technology of the People's Republic of China (2015CB553903), and the research-oriented clinician funding program of Tongji Medical College, Huazhong University of Science and Technology. The funders played no role in the study design, data collection and analysis, decision to publish, or preparation of the manuscript.

\section{Contributors}

Peng Wu, Yi Wang and Yifan Meng conceived and designed the study; Yi Wang and Wending $\mathrm{Li}$ performed the statistical analysis; Yifan Meng, Yi Wang and Wending Li drafted the article; Xiaofei Zhang, Zaixing Deng, Mengjun Hu, Pingrong Shen, Shengfeng $\mathrm{Xu}$, Chenglin $\mathrm{Fu}$, Wen Jiang, Bing $\mathrm{Wu}$, Kezhen Li, Ling Xi, Ding Ma, Xing Xie, Junbo Hu, Xiaodong Cheng contributed to the data collection and quality control; Peng $\mathrm{Wu}$ made the critical revision of the manuscript. All authors gave their comments on the article and approved the final version before submission.

\section{Competing Interests}

The authors have declared that no competing interest exists.

\section{References}

1. Denny L, Herrero R, Levin C, Kim JJ. Cervical Cancer. In: Gelband H, Jha P, Sankaranarayanan R, Horton S, editors. Cancer: Disease Control Priorities, Third Edition (Volume 3). Washington (DC); 2015.

2. Bouvard V, Baan R, Straif K, Grosse Y, Secretan B, El Ghissassi F, et al. A review of human carcinogens--Part B: biological agents. Lancet Oncol. 2009; 10: 321-2.

3. Bruni L, Diaz M, Castellsague X, Ferrer E, Bosch FX, de Sanjose S. Cervical human papillomavirus prevalence in 5 continents: meta-analysis of 1 million women with normal cytological findings. J Infect Dis. 2010; 202: 1789-99.

4. Zeng Z, Yang H, Li Z, He X, Griffith CC, Chen X, et al. Prevalence and Genotype Distribution of HPV Infection in China: Analysis of 51,345 HPV Genotyping Results from China's Largest CAP Certified Laboratory. J Cancer. 2016; 7: 1037-43.

5. Lu JF, Shen GR, Li Q, Chen X, Ma CF, Zhu TH. Genotype distribution characteristics of multiple human papillomavirus in women from the Taihu River Basin, on the coast of eastern China. BMC Infect Dis. 2017; 17: 226.

6. Wang R, Guo XL, Wisman GB, Schuuring E, Wang WF, Zeng ZY, et al. Nationwide prevalence of human papillomavirus infection and viral genotype distribution in 37 cities in China. BMC Infect Dis. 2015; 15: 257. 
7. Tang Y, Zheng L, Yang S, Li B, Su H, Zhang LP. Epidemiology and genotype distribution of human papillomavirus (HPV) in Southwest China: a cross-sectional five years study in non-vaccinated women. Virol J. 2017; 14: 84.

8. Zhang Y, Wang Y, Liu L, Guo C, Liu Z, Nie S. Prevalence of human papillomavirus infection and genotyping for population-based cervical screening in developed regions in China. Oncotarget. 2016; 7: 62411-24.

9. Li Z, Liu F, Cheng S, Shi L, Yan Z, Yang J, et al. Prevalence of HPV infection among 28,457 Chinese women in Yunnan Province, southwest China. Sci Rep. 2016; 6: 21039

10. Ma X, Wang Q, Ong JJ, Fairley CK, Su S, Peng P, et al. Prevalence of human papillomavirus by geographical regions, sexual orientation and HIV status in China: a systematic review and meta-analysis. Sex Transm Infect. 2018; 94(6):434-42.

11. [Internet] China Food and Drug Administration. Human papillomavirus (HPV) nucleic acid detection and genotype reagent technology guidelines for review. http://app2.sfda.gov.cn/datasearchp/gzcxSearch.do?formRender $=$ cx\&page $=1$

12. Day SP, Hudson A, Mast A, Sander T, Curtis M, Olson S, et al. Analytical performance of the Investigational Use Only Cervista HPV HR test as determined by a multi-center study. J Clin Virol. 2009; 45 Suppl 1: S63-72.

13. Xiao SS, Fan JL, He SL, Li YR, Wang LY, Yu KN, et al. Analysis of Human Papillomavirus Infection in 16,320 Patients From a Gynecology Clinic in Central South China. J Low Genit Tract Dis. 2016; 20: 327-31.

14. Zeng Z, Austin RM, He X, Chen X, Guo X, Zheng B, et al. Prevalence of High-Risk Human Papillomavirus Infection in China: Analysis of 671,163 Human Papillomavirus Test Results From China's Largest College of American Pathologists-Certified Laboratory. Am J Clin Pathol. 2016; 145: 622-5.

15. Franceschi S, Denny L, Irwin KL, Jeronimo J, Lopalco PL, Monsonego J, et al. Eurogin 2010 roadmap on cervical cancer prevention. Int J Cancer. 2011; 128: 2765-74.

16. Huh WK, Ault KA, Chelmow D, Davey DD, Goulart RA, Garcia FA, et al. Use of primary high-risk human papillomavirus testing for cervical cancer screening: interim clinical guidance. Gynecol Oncol. 2015; 136: 178-82.

17. Saslow D, Solomon D, Lawson HW, Killackey M, Kulasingam SL, Cain J, et al. American Cancer Society, American Society for Colposcopy and Cervical Pathology, and American Society for Clinical Pathology screening guidelines for the prevention and early detection of cervical cancer. Am J Clin Pathol. 2012; 137: 516-42.

18. Liu SS, Chan KY, Leung RC, Chan KK, Tam KF, Luk MH, et al. Prevalence and risk factors of Human Papillomavirus (HPV) infection in southern Chinese women - a population-based study. PLoS One. 2011; 6: e19244.

19. Zhao FH, Tiggelaar SM, Hu SY, Xu LN, Hong Y, Niyazi M, et al. A multi-center survey of age of sexual debut and sexual behavior in Chinese women: suggestions for optimal age of human papillomavirus vaccination in China. Cancer Epidemiol. 2012; 36: 384-90.

20. Syrjanen K, Kulmala SM, Shabalova I, Petrovichev N, Kozachenko V, Zakharova $\mathrm{T}$, et al. Epidemiological, clinical and viral determinants of the increased prevalence of high-risk human papillomavirus (HPV) infections in elderly women. Eur J Gynaecol Oncol. 2008; 29: 114-22.

21. National Health and Family Planning Commission of the PRC. Statistics yearbook of health and family planning in China: Chinese Peking Union Medical College Press; 2016.

22. Yu J. Teenage sexual attitudes and behaviour in China: a literature review. Health Soc Care Community. 2012; 20: 561-82.

23. Sureshkumar BT, Shanmughapriya S, Das BC, Natarajaseenivasan K. A population-based study of the prevalence of HPV in three districts of Tamil Nadu, India. Int J Gynaecol Obstet. 2015; 129: 58-61.

24. Hildesheim A, Wang SS. Host and viral genetics and risk of cervical cancer: a review. Virus Res. 2002; 89: 229-40.

25. Zhao J, Du H, Belinson JL, Qu X, Zhang W, Mei J, et al. Evaluation of The Cervista HPV A9 group In Screening Patients for Cervical Cancer. J Med Screen. 2016; 23: 38-43.

26. Poljak M. Prophylactic human papillomavirus vaccination and primary prevention of cervical cancer: issues and challenges. Clin Microbiol Infect. 2012; 18 Suppl 5: 64-9.

27. Schiffman M, Solomon D. Clinical practice. Cervical-cancer screening with human papillomavirus and cytologic cotesting. N Engl J Med. 2013; 369: 2324-31. 